\title{
Limits of steady burning propellants in the phenomenological theory using effective initial temperature
}

\author{
Kanysh O. SABDENOV*, Johann DUECK ** and Erzada MAIRA* \\ *L.N. Gumilyov Eurasian National University \\ Munaitpasov-Str. 5, 010008 Astana, Kazakhstan \\ E-mail:mayira76@yahoo.co.jp \\ ${ }^{* *}$ Friedrich-Alexander-Universität Erlangen-Nuremberg \\ Paul-Gordan-Str. 3 \\ 91052 Erlangen, Germany
}

Received 7 September 2014

\begin{abstract}
A new form of the phenomenological theory of unsteady combustion of solid propellants is proposed. It is based on the explicit use of a single physical quantity - effective initial combustion temperature. The solution of the problem of combustion stability in the presence of heat loss from the side surface of fuel of cylindrical form is given. The heat loss reduces the region of combustion stability and can lead to extinction.
\end{abstract}

Key words : Solid propellant transient burning, Combustion stability condition, Phenomenological theory of unsteady combustion, Effective initial temperature

\section{Introduction}

Theoretical study of unsteady combustion of solid propellants began mainly in two directions.

In the first, researchers developed the models for gasification of solid fuel and flame pattern in the gas phase (Denison, M.R., and Baum, E.A., 1961, Krier, H., T'ien, J.S., Sirignano, W.A., and Summerfield, M., 1968, Beckstead, M.W., Derr, R.L., and Price, C.F., 1970). To develop the models of combustion here requires detailed knowledge of the kinetics of chemical reactions, mechanisms of complex phase transformations, etc... This is a laborious way, great difficulties arise in the description of multi-component chemical reactions. But detailed modelling allows to retrieve a large amount of information about the combustion of rocket fuels, including effects such as the influence of the flow conditions of the fuel gas flow under a semi-closed chamber (Mukunda, H.S., 1978, Greatrix, D. R., 2007, Srinivasan, K., Narayanan, S., and Sharma, O.P., 2008). In addition, there are no restrictions on the time characteristics of possible transient phenomena (Roh, T.-S., Tseng, I.-S., and Yang, V., 1995, Apte, S., and Yang, V., 2002, Cai, W., Ma, F., and Yang, V., 2003, Willcox, M.A., Brewster, M.Q., Tang, K.C., Stewart, D.S., and Kuznetzov, I., 2007). Such models are relatively free in describing the various structures of propellant and influences of external factors (Wang, X., Jackson, T.L., and Massa, L., 2004, Beckstead, M.W., Puduppakkam, K., Thakre, P., and Yang, V., 2007).

In the works of the second direction the detailed description of the mechanism of transformation of the solid phase fuel to the combustion products is not required. Only a number of experimental dependences of steady state combustion is need to know. Therefore, this trend has been called the phenomenological approach, and itself a theoretical construct is known as the phenomenological theory of unsteady combustion Zeldovich - Novozhilov (ZN-theory) (Zeldovich, Ya. B., 1942, Novozhilov, B.V., 1973, Novozhilov, B.V., 1990). Applicability of the theory ZN is restricted by frequencies transient phenomena, since it is assumed that the characteristic time of non-stationary processes is not less than the thermal relaxation time of the surface layer of propellant.

Attempts to empower the phenomenological approach to high frequencies (Vilyunov, V.N., and Sabdenov, K.O., 1992) found a number of difficulties. First of all it is the lack of reliable grounds for applying the experimental 
dependences obtained for stationary combustion mode. Theoretical scheme proposed by (Vilyunov, V.N., and Sabdenov, K.O., 1992) allows formally exclude detailed modelling of the kinetics of chemical reactions. This can be done both for the gasification of solid fuel and for the flame in the gas phase. But the using of experimental burning rate dependences of the temperature and pressure under conditions of nonstationarity is not strictly justified. This matter requires further consideration.

Study of erosive burning (under blowing) can also be performed without considering detailed kinetics of chemical reactions (Sabdenov, K.O., 1996, Sabdenov, K.O., 2013, Sabdenov, K.O., 2008, Sabdenov, K.O., and Erzada Maira, 2013. Therefore, the effect of erosion effects on unsteady combustion can be taken into account in a phenomenological approach.

There are other problems in the phenomenological approach, they are discussed in detail by (Zarko, V.E., and Gusachenko, L.K., 2010). Its further development must be seen not only in dealing with specific applications, but also in the critical revision of the foundations and theoretical proposals. For further discussion of the material below, we first briefly discuss the fundamental aspects of ZN-theory.

\section{Bases of ZN-theory}

ZN-theory is based on the following arguments, which are based on two experimental facts. The first states the dependence of the stationary combustion velocity $u^{0}$ on the initial temperature $T_{0}$ and pressure $p^{0}$ :

$$
u^{0}=u^{0}\left(T_{0}, p^{0}\right) \text {. }
$$

Here and below the upper-index 0 means the stationary values.

The second fact reflects the surface temperature gasification of solid fuel $T_{\mathrm{s}}^{0}$ on the same variables:

Obviously, from the equations (1) and (2) must be

$$
T_{\mathrm{s}}^{0}=T_{\mathrm{s}}^{0}\left(T_{0}, p^{0}\right) \text {. }
$$

$$
u^{0}=u^{0}\left(T_{\mathrm{s}}^{0}, p^{0}\right)
$$

Thermal relaxation time in the solid phase of propellant is much longer than the relaxation time of diffusion-thermal and hydrodynamic processes in gas phase. This allows to consider only the thermal processes in the solid phase of propellant for relatively slow variations in temperature, burning rate and other dynamic variables.

Let the flame front moves from right to left along the coordinate $x$. Then, in a moving coordinate system associated with the combustion front, the temperature distribution in the solid phase $T_{\mathrm{c}}$ can be described by the equation of heat conduction

$$
\frac{\partial T_{\mathrm{c}}}{\partial t}+u \frac{\partial T_{\mathrm{c}}}{\partial x}=\kappa_{\mathrm{c}} \frac{\partial^{2} T_{\mathrm{c}}}{\partial x^{2}},
$$

where are: $t$ - time; $u$ - transient burning rate of propellant; $\kappa_{\mathrm{c}}=\lambda_{\mathrm{c}} /\left(\rho_{\mathrm{c}} c_{\mathrm{c}}\right) ; \rho_{\mathrm{c}}, \lambda_{\mathrm{c}}, c_{\mathrm{c}}-$ density, thermal conductivity and heat capacity of the fuel. Surface decomposition of the fuel is at the point $x=0$, and the fuel itself occupies the region $x<0$.

Further is assumed that the density $\rho_{\mathrm{c}}$, the thermal conductivity $\lambda_{\mathrm{c}}$ and the heat capacity $c_{\mathrm{c}}$ are constant in magnitude.

For the case of steady state combustion:

$$
u^{0} \frac{d T_{\mathrm{c}}^{0}}{d x}=\kappa_{\mathrm{c}} \frac{d^{2} T_{\mathrm{c}}^{0}}{d x^{2}} .
$$

This equation under considering the boundary conditions

$$
T_{\mathrm{c}}^{0}(-\infty)=T_{0}, \quad T_{\mathrm{c}}^{0}(0)=T_{\mathrm{s}}^{0},
$$

leads to the following temperature profile in the solid phase:

$$
T_{\mathrm{c}}^{0}=T_{0}+\left(T_{\mathrm{s}}^{0}-T_{0}\right) \cdot \exp \left(u^{0} x / \kappa_{\mathrm{c}}\right)
$$

Stationary temperature gradient $f^{0}$ on the burning surface is given as follows from (6), as the expression

$$
f^{0}=\left.\frac{d T_{\mathrm{c}}^{0}}{d x}\right|_{x=0}=\frac{u^{0}\left(T_{\mathrm{s}}^{0}-T_{0}\right)}{\kappa_{\mathrm{c}}} .
$$

Expressing from (7) the initial temperature $T_{0}=T_{\mathrm{s}}^{0}-\kappa_{\mathrm{c}} f^{0} / u^{0}$, we rewrite (1) and (2) as follows:

$$
u^{0}=u^{0}\left(T_{\mathrm{s}}^{0}-\kappa_{\mathrm{c}} f^{0} / u^{0}\right), \quad T_{\mathrm{s}}^{0}=T_{\mathrm{s}}^{0}\left(T_{\mathrm{s}}^{0}-\kappa_{\mathrm{c}} f^{0} / u^{0}\right) .
$$

Two boundary conditions (5) are not enough to determine the thermal state of the propellant under combustion in the transient regime. It is necessary to specify the value of the heat flux at the moving boundary $(x=0)$ between the 
solid and gas phases.

Boundaries mobility is not explicitly visible, but its presence reflects the dependence of the burning rate $u(t)$ and the surface temperature of the fuel $T_{\mathrm{s}}(t)$ from time.

Solution to the problem of formulation of additional boundary conditions in ZN-theory is taking of (8), where arguments $T_{\mathrm{s}}$ and $f$ are variables (here and further $p=$ const):

$$
u=u\left(T_{\mathrm{s}}-\kappa_{\mathrm{f}} f / u\right), \quad T_{\mathrm{s}}=T_{\mathrm{s}}\left(T_{\mathrm{s}}-\kappa_{\mathrm{f}} / u\right),
$$

with non-stationary temperature gradient $f=\left(T_{\mathrm{s}}-T_{0}{ }^{*}\right) u / \kappa_{\mathrm{c}}$. The explanation for the temperature $T_{0}{ }^{*}$ is given below.

Then equation (4), (9) represent a full statement of the problem of unsteady combustion of solid propellants. They are valid for the cases of non-stationary processes with relaxation times of the order of warm-up time in the solid phase of fuel $t_{\mathrm{c}}=\kappa_{\mathrm{c}} /\left(u^{0}\right)^{2}$.

The main advantage of $\mathrm{ZN}$-theory is the need for the minimum information about the kinetics of complex chemical reactions.

The phenomenological theory of Zel'dovich - Novozhylov uses the concept of effective initial temperature of propellant. But it serves only as an intermediary to substantiate the possibility of application to non-stationary processes of experimental data of stationary burning rate and surface temperature of propellant. The effective initial temperature in $\mathrm{ZN}$-theory plays a secondary role, in fact, it is excluded by defining it as an effective initial temperature $T_{0}{ }^{*}=T_{\mathrm{s}}-\kappa_{\mathrm{c}} f / u$, which is considered applicable for non-stationary conditions (Novozhilov, B.V., 1974).

Thus, this temperature is replaced by its gradient at the surface $f$, which in the future plays a leading role in the description of non-stationary phenomena. This means that all results obtained within the framework of ZN-theory should find a physical justification on the basis of variable temperature gradient.

The formulation of the equations (9) admits some criticisms. First, the use of (9) for solving unsteady combustion by analytical methods leads to cumbersome calculations. Second, difficulties arise when considering the combustion at very low temperatures or pressures, when combustion becomes sensitive to perturbations leading to extinction, or when the propellant simply can not burn in layers.

A similar remark applies to the cases of the burning reaction at higher temperatures or pressures. In these cases, values of $T_{0}{ }^{*}$ (of course, as well as pressure) may be beyond the range of experimental use of the initial temperature, and hence, the results lose the credibility.

\section{3. $T_{0}{ }^{*}$-theory}

Below is shown, how the phenomenological approach can be reformulated (as proposed by Sabdenov K.O., 2013), abandoning from the use of effective initial temperature after Zel'dovich / Novozhilov.

In the $T_{0}{ }^{*}$-theory the new definition of the effective initial temperature $T_{0}{ }^{*}$ is used. Here the basic relations under unsteady combustion mode are written as

$$
u=u\left(T_{0}^{*}\right), \quad T_{\mathrm{s}}=T_{\mathrm{s}}\left(T_{0}^{*}\right)
$$

i.e. in comparison with (1) and (2) $T_{0}$ is replaced by $T_{0}{ }^{*}$. In other words, it is assumed that in an unsteady combustion mode there is an effective initial temperature $T_{0}{ }^{*}(t)$, which is different from $T_{0}$. Burning rate and surface temperature gasification fuel can be calculated from the experimental dependences (1) and (3), but with the temperature $T_{0}^{*}$ corresponding to (10).

In the same way as in the $\mathrm{ZN}$-theory here to determine the burning rate requires a knowledge of the heat flux $\lambda_{\mathrm{c}} f$ at the interface between the solid and gas phases.

This boundary condition can be formulated as follows: suppose that the formula for the gradient in the stationary combustion mode is also valid for transient condition, if the calculation of the burning rate $u$ and the surface temperature $T_{\mathrm{s}}$ is performed at the time-dependent effective initial temperature $T_{0}{ }^{*}(t)$. Thus (Zeldovich, Ya. B., 1942, Novozhilov, B.V., 1973, Novozhilov, B.V., 1990),

$$
\left.\lambda_{\mathrm{c}} \frac{\partial T_{\mathrm{c}}}{\partial x}\right|_{x=0}=c_{\mathrm{c}} \rho_{\mathrm{c}}\left(T_{\mathrm{s}}-T_{0}^{*}\right) \cdot u \text {, where } u=u\left(T_{0}^{*}\right)=u\left(T_{\mathrm{s}}\right) .
$$

The effective initial temperature $T_{0}{ }^{*}$ can be determined by one of the experimental curves (1) or (2), considering them to be valid for non-stationary combustion mode using the known values $u$ or $T_{\mathrm{s}}: u=u\left(T_{0}{ }^{*}\right), T_{\mathrm{s}}=T_{\mathrm{s}}\left(T_{0}{ }^{*}\right)$.

Fig. 1 shows a graphical method for determining $T_{0}{ }^{*}$ using the second formula.

On the curve $T_{\mathrm{s}}^{0}=T_{\mathrm{s}}^{0}\left(T_{0}\right)$, (in the Kelvin temperature), reliable physical meaning has only the part shown by the solid line, which is obtained empirically. It suggests the possibility of stationary combustion in a limited range of 
variation of the initial temperature.

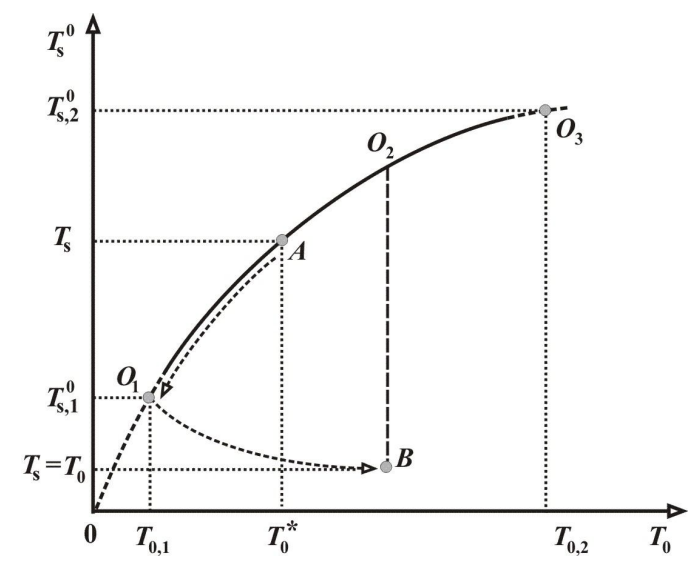

Fig. 1. Curve for $T_{\mathrm{s}}^{0}=T_{\mathrm{s}}{ }^{0}\left(T_{0}\right)$ and determination using it $T_{0}{ }^{*}$ by the known value $T_{\mathrm{s}}$

With dashed lines, continuing the curve towards low and high initial temperatures, are shown extrapolated areas. They can also indicate stationary combustion modes, but there is no reliable information about them because of lack of the reliable experimental data.

The lower $O_{1}$ and upper $O_{3}$ boundaries correspond to low and high temperature limits of the propagation of the flame front. The point $A$ in the nonstationary combustion mode moves along the curve $O_{1} O_{3}$.

It is not excluded that when solving the problem of unsteady combustion, we can go outside the area $O_{1} O_{3}$. Then, presumably, the theoretical results obtained are likely to be erroneous or extremely rude. But most probable scenario is the following.

Going beyond the lower boundary $O_{1}$, point $A$ will continue to move to point $B$ along the curve $O_{1} B$. This means occurrence of extinction: $T_{\mathrm{s}} \rightarrow T_{0}, T_{0}{ }^{*} \rightarrow T_{0}$.

This interpretation is consistent with data obtained from the analysis of unsteady combustion based on a detailed description of the gas phase and chemical reaction in the fuel (Sabdenov, K.O., 2013). Therefore, transient form $u\left(T_{0}{ }^{*}\right.$ ) must be supplemented by the condition: $u\left(T_{0}{ }^{*}\right)=0$, if $T_{0}{ }^{*}<T_{0,1}$, that is a reflection of experimental fact (Novozhilov, B.V., 1974) about impossibility of burning at lower initial temperature of propellant below the critical value $T_{0,1}$ (Fig. 1).

From the presented scenario it seems that combustion is similar to steady-state combustion at low temperatures. But actually, this obviously does not physically occur.

The seeming strong decrease of the initial temperature is simply a reflection a violation of the heat balance in the reaction zone under unsteady combustion, leading to the cessation of burning. And when it happens, no internal mechanisms in the system are able to initiate combustion again.

Thus, the condition of extinction in $T_{0}{ }^{*}$-theory is not determined by the temperature gradient on the surface of the fuel, but only by temperature $T_{0}{ }^{*}$ on the experimental curve $T_{\mathrm{s}}^{0}\left(T_{0}\right)$ (Fig. 1). This is the main difference between $T_{0}{ }^{*}$-theory and $\mathrm{ZN}$-theory.

Transition beyond the upper limit, where $T_{0}{ }^{*}>T_{0,2}, T_{0,2}$ - the upper critical temperature, would mean under steady state conditions the transition from front to ignition combustion (thermal explosion) of the entire sample of propellant, i.e. is virtually impossible to form the combustion wave.

Then follows the second condition $u\left(T_{0}{ }^{*}\right)=\infty$, if $T_{0}{ }^{*}>T_{0,2}$.

Here, the symbol of infinity does not introduce meaningless physical content: thermal explosion can be interpreted as a frontal burning with infinite velocity flame.

Each limit value of the initial temperature $T_{0,1}$ and $T_{0,2}$ correspond to the limit temperature $T_{\mathrm{s}, 1}{ }^{0}$ and $T_{\mathrm{s}, 2}{ }^{0}$ (Fig. 1).

Accurate and complete data on the values of the critical temperature $T_{0,1}$ and $T_{0,2}$ are unavailable. But the existing experimental data indicates the following approximate estimates (Novozhilov, B.V., 1974): $T_{0,1} \sim 100 \ldots 150 \mathrm{~K}, \quad T_{0,2} \sim$ $650 \ldots 700 \mathrm{~K}$.

As for the part $\mathrm{O}_{2} \mathrm{O}_{3}$, then due to the lack of experimental data on it, the situation is uncertain. On this section may 
be both stationary and non-stationary (e.g. oscillatory) combustion modes, as well as at the site near the point $O_{1}$.

It should be noted that the effective initial temperature principle can not be measured, the sole exception is the stationary combustion mode when $T_{0}{ }^{*}=T_{0}$.

All the arguments above were carried out for a fixed base pressure $p_{0}$. If the pressure is variable, then for each value of a set of $p_{01}, p_{02}, p_{03}, \ldots$ has its own curve, similar to that shown in Fig. 1. Therefore unsteady combustion mode with varying pressure $p(t) A$ point will jump from one curve to another curve of this family.

Overall physical picture of burning is highly complicated and is not discussed here. But it is clear that the regime of unsteady combustion should be considered on a two-dimensional surface: $T_{\mathrm{s}}\left(T_{0}{ }^{*}, p\right)$ or $u\left(T_{0}{ }^{*}, p\right)$.

In addition with critical conditions to temperature similar conditions to pressure appear, containing an upper $p_{0,2}$ and lower $p_{0,1}$ borders. The existence of $p_{0,1}$ in theory is dictated by experimental fact about impossibility of burning propellants at very low pressures (Novozhilov, B.V., 1974). As for the upper value of $p_{0,2}$, this issue still has not been studied in detail. Most likely, it would be comparable to the pressure which is realized in the front of the detonation wave (if combustion is possible for this type of fuel).

Introduction of critical temperature $T_{0,1}, T_{0,2}$ and pressures $p_{0,1}, p_{0,2}$ is a natural step. This corresponds to the idea of the phenomenological approach, where all the necessary information for the theory is taken from of experiment. The existence and the numerical values of such critical parameters can not be set while remaining within the phenomenological approach. Only with theories that are based on knowledge of the detailed kinetics of chemical processes, critical parameters can be identified and calculated theoretically.

Already Novozhilov B.V. (Novozhilov, B.V., 1990) indicates the possible uncertainty of the theoretical results in the case of withdrawal effective initial temperature beyond the capabilities of the experimental curves (1)-(3).

The weakest point in ZN-theory is the determination of $T_{0}{ }^{*}$. Previously by other authors (Zarko, V.E., and Kiskin, A.B., 1980, Zarko, V.E., Simonenko, V.N., and Kiskin, A.B., 1987) was proposed to determine the temperature from the first equation of (10). But in $T_{0}^{*}$-theory the interval of effective initial temperature obeys to strict limitations.

So involvement (11) is caused by the need to eliminate the incompleteness in physical and mathematical formulation of the problem of unsteady combustion.

For stationary combustion such difficulties do not arise. In the coordinate system associated with the combustion front, the boundary is fixed. Boundary condition (11) at steady state is performed automatically.

Thus, in full view the mathematical problem of unsteady combustion (in the absence of additional heat sources or sinks) is formulated as follows:

$$
\frac{\partial T_{\mathrm{c}}}{\partial t}+u \frac{\partial T_{\mathrm{c}}}{\partial x}=\kappa_{\mathrm{c}} \frac{\partial^{2} T_{\mathrm{c}}}{\partial x^{2}}
$$

$u=u\left(T_{0}{ }^{*}, p\right), \quad T_{\mathrm{s}}=T_{\mathrm{s}}\left(T_{0}{ }^{*}, p\right)$.

Boundary conditions:

$x=-\infty: \quad T_{\mathrm{c}}=T_{0}$

$x=0: \quad T_{\mathrm{c}}=T_{\mathrm{s}}, \quad \frac{\partial T_{\mathrm{c}}}{\partial x}=\frac{u\left(T_{0}^{*}, p\right)\left(T_{\mathrm{s}}-T_{0}^{*}\right)}{\kappa_{\mathrm{c}}}$.

Condition of limits:

$$
\begin{aligned}
& u\left(T_{0}{ }^{*}, p\right)=0, \quad \text { if } \quad T_{0}{ }^{*}<T_{0,1} \quad \text { or } \quad p<p_{0,1} \\
& u\left(T_{0}{ }^{*}, p\right)=\infty, \quad \text { if } \quad T_{0}{ }^{*}>T_{0,2} \quad \text { or } \quad p>p_{0,2} .
\end{aligned}
$$

The initial conditions to the system (12) will be taken

$$
T_{\mathrm{c}}(0, x)=T_{\mathrm{c}}^{0}(x), \quad u^{0}\left(T_{0}\right)=u^{0}\left(T_{0}, p_{0}\right), \quad T_{\mathrm{s}}^{0}=T_{\mathrm{s}}^{0}\left(T_{0}, p_{0}\right),
$$

as the most important tasks for the practice are to study the deviations from the stationary combustion mode.

Thus, the main idea in a phenomenological approach is the introduction of the concept and operation of an effective initial temperature $T_{0}{ }^{*}$, in which the combustion occurs under the unsteady conditions with a surface temperature $T_{0}{ }^{*}$, and which allows recalculate the new states of the nonstationary case from the experimental dependences for stationary combustion (1) - (3).

It should be noted that the dependence (1) is easy to determine experimentally. The other two formulas (2) and (3) are difficult to establish because of the difficulty of measuring the temperature $T_{\mathrm{s}}^{0}$. The complexity of determining the dependence $T_{\mathrm{s}}^{0}\left(T_{0}\right)$ does not detract from the value of the theory - it's just technical problem.

Now a solution of a particular problem will be given below within the framework of the theory presented. This is the determination of limits of stable combustion with strong heat loss from the side surface of the cylindrical propellant 
charge.

\section{Stability burning of propellant under conditions of heat loss. Linear Approximation}

Condition for stable combustion of propellants when there are large heat losses from the lateral surface of the cylindrical sample, is of interest for the operation of small rocket engines.

These motors can be used for the orientation and manoeuvring for small missiles weighing about $100 \mathrm{~kg}$ and less (Sandau R., Roeser H.-P., and Valenzuela A., 2008, The Role of Small Satellites in NASA and NOAA Earth Observation Programs, 2000).

Let the heat flux $j_{\mathrm{s}}$ from the lateral surface propellant sample be equal to

$$
j_{\mathrm{s}}=\alpha\left(T_{\mathrm{c}}-T_{0}\right),
$$

where $\alpha$-is a heat transfer coefficient. The $x$ axis is directed along the length of the cylinder of radius $R_{\mathrm{c}}$. Then the combustion front can be described by the equation

$$
\frac{\partial T_{\mathrm{c}}}{\partial t}+u \frac{\partial T_{\mathrm{c}}}{\partial x}=\kappa_{\mathrm{c}} \frac{\partial^{2} T_{\mathrm{c}}}{\partial x^{2}}-\frac{2 \alpha}{c_{c} \rho_{c} R_{c}}\left(T_{c}-T_{0}\right) .
$$

Further are used dimensionless quantities: temperature $\theta_{\mathrm{c}}=\left(T_{\mathrm{c}}-T_{0}\right) /\left(T_{\mathrm{s}}^{0}-T_{0}\right)$, the burning rate $B=u / u^{0}$, surface temperature $\theta_{\mathrm{s}}=\left(T_{\mathrm{s}}-T_{0}\right) /\left(T_{\mathrm{s}}^{0}-T_{0}\right)$, time $\tau=t\left(u^{0}\right)^{2} / \kappa_{\mathrm{c}}$ and coordinate $\xi=x u^{0} / \kappa_{\mathrm{c}}$.

Equation (14) in these new variables becomes the form:

$$
\frac{\partial \theta_{\mathrm{c}}}{\partial \tau}+B \frac{\partial \theta_{\mathrm{c}}}{\partial \xi}=\frac{\partial^{2} \theta_{\mathrm{c}}}{\partial \xi^{2}}-\varphi \theta_{\mathrm{c}}, \quad \varphi=\frac{2 \alpha}{c_{\mathrm{c}} \rho_{\mathrm{c}} R_{\mathrm{c}}} \frac{\kappa_{\mathrm{c}}}{\left(u^{0}\right)^{2}} .
$$

Stationary solution $\theta_{\mathrm{c}}^{0}(\xi)$ of this equation must satisfy the boundary conditions

They are equivalent to the conditions (13).

$$
\theta_{\mathrm{c}}^{0}(-\infty)=0, \quad \theta_{\mathrm{c}}^{0}(0)=1, \quad B=1 .
$$

A simple calculation gives

$$
\theta_{\mathrm{c}}^{0}(\xi)=\exp (\chi \xi), \quad \chi=\frac{1+\sqrt{1+4 \varphi}}{2} .
$$

Then, in the steady state on the burning surface $x=0$ is valid

$$
\frac{\partial T_{\mathrm{c}}^{0}}{\partial x}=f^{0}=\frac{\chi u^{0}}{\kappa_{c}}\left(T_{s}^{0}-T_{0}\right) .
$$

According to $T_{0}{ }^{*}$-theory under unsteady conditions, equation (15) must be supplemented by the conditions

$$
\theta_{\mathrm{c}}(-\infty, \tau)=0, \quad \theta_{\mathrm{c}}(0, \tau)=\theta_{\mathrm{s}}=\theta_{\mathrm{s}}\left(\theta_{0}{ }^{*}\right), \quad B=B\left(\theta_{0}^{*}\right),\left.\quad \frac{\partial \theta_{\mathrm{c}}}{\partial \xi}\right|_{\xi=0}=\chi B \cdot\left(\theta_{s}-\theta_{0}^{*}\right),
$$

where the dimensionless effective initial temperature is $\theta_{0}{ }^{*}=\left(T_{0}{ }^{*}-T_{0}\right) /\left(T_{\mathrm{s}}^{0}-T_{0}\right)$.

So far we do not engage the restrictions terms.

Within the framework of the linear stability theory the task is to analyze the behaviour of the solution of equation (15) with conditions (17) in a small neighbourhood of the stable combustion, expressed by (16).

Unsteady solution of (15) is sought in the form

$$
\theta_{\mathrm{c}}=e^{\chi \xi}+\vartheta(\xi) e^{\Omega \tau}, \quad B=1+b e^{\Omega \tau},
$$

where $\vartheta(\xi), b$ are small perturbations of temperature and combustion rate.

Their use in (15) and keeping only the linear values leads to the equation

$$
\frac{d^{2} \vartheta}{d \xi^{2}}-\frac{d \vartheta}{d \xi}-(\varphi+\Omega) \vartheta=b \frac{d \theta_{\mathrm{c}}^{0}}{d \xi} .
$$

Its solution corresponding to the condition $\vartheta(-\infty)=0$, has the form

$$
\vartheta(\xi)=A \cdot e^{q \xi}-\frac{\chi b}{\Omega} \cdot e^{\chi \xi}, \quad q=\frac{1+\sqrt{1+4(\varphi+\Omega)}}{2} .
$$

We consider the last two terms in (17). Let $\delta \theta_{\mathrm{s}}$ to be a small perturbation of the surface temperature of decomposition of the propellant. Up to small quantities of order $\left(\delta \theta_{\mathrm{s}}\right)^{2}$ for the combustion rate and gradient can be 
assumed

$$
B \approx 1+\frac{d B}{d \theta_{\mathrm{s}}} \delta \theta_{\mathrm{s}}=1+\frac{d B}{d \theta_{0}^{*}} \frac{d \theta_{0}^{*}}{d \theta_{\mathrm{s}}} \delta \theta_{\mathrm{s}}=1+\frac{k}{r} \delta \theta_{\mathrm{s}} .
$$

Here, $k$ and $r$ are phenomenological coefficients (Novozhilov, B.V., 1974, Vilyunov, V.N., and Sabdenov, K.O., 1992):

$$
k=\frac{d B}{d \theta_{0}^{*}} \approx \frac{T_{s}^{0}-T_{0}}{u^{0}} \frac{d u^{0}}{d T_{0}}, \quad r=\frac{d \theta_{\mathrm{s}}}{d \theta_{0}^{*}} \approx \frac{d T_{s}^{0}}{d T_{0}} .
$$

These coefficients are obtained from the experiment. Analogues can be get

$$
\begin{aligned}
\frac{\partial \theta_{\mathrm{c}}}{\partial \xi} \approx \chi\left(1+\frac{k}{r} \delta \theta_{\mathrm{s}}\right) \cdot\left[1+\delta \theta_{\mathrm{s}}\right. & \left.-\left(0+\delta \theta_{0}^{*}\right)\right]= \\
& =\chi\left(1+\frac{k}{r} \delta \theta_{\mathrm{s}}\right) \cdot\left[1+\delta \theta_{\mathrm{s}}-\frac{d \theta_{0}^{*}}{d \theta_{\mathrm{s}}} \delta \theta_{\mathrm{s}}\right] \approx \chi+\chi \frac{k+r-1}{r} \delta \theta_{\mathrm{s}} .
\end{aligned}
$$

Next, we set the left-hand sides of equations (19) and (20) as follows

$$
B=1+b e^{\Omega \tau}, \quad \frac{\partial \theta_{\mathrm{c}}(\tau, 0)}{\partial \xi}=\left(\frac{d \theta_{\mathrm{c}}^{0}}{d \xi}+e^{\Omega \tau} \frac{d \vartheta_{\mathrm{c}}}{d \xi}\right)_{\xi=0}=\chi+\frac{d \vartheta_{\mathrm{c}}(0)}{d \xi} \cdot e^{\Omega \tau}
$$

Then in view of equality $\delta \theta_{\mathrm{s}}=\vartheta_{\mathrm{c}}(0) e^{\Omega \tau}$ the equations (19), (20) become the form

$$
b=\frac{k}{r} \vartheta_{\mathrm{c}}(0), \quad \frac{d \vartheta_{\mathrm{c}}(0)}{d \xi}=\chi a \vartheta_{\mathrm{c}}(0), \quad a=\frac{k+r-1}{r} .
$$

The use here the formulas (18) after simple transformations gives a system of algebraic equations for the unknowns $A, b$ :

$$
\begin{gathered}
A-\left(\frac{r}{k}+\frac{\chi}{\Omega}\right) b=0, \\
A(q-\chi a)+\frac{\chi^{2}}{\Omega} \frac{k-1}{r} b=0 .
\end{gathered}
$$

This homogeneous system has a nontrivial solution if its determinant $\Delta_{0}$ is zero:

$$
\Delta_{0}=(q-\chi a)\left(\frac{\chi}{\Omega}+\frac{r}{k} \Omega\right)+\frac{k-1}{r} \chi^{2}=0
$$

Dropping simple but somewhat cumbersome intermediate transformations, we note that such a requirement $\Delta_{0}=0$ leads to an equivalent characteristic equation for the increment $\Omega$ :

$$
\begin{gathered}
\Omega^{2}+\frac{\Lambda}{r^{2}} \Omega+\frac{k \chi^{2}}{r^{2}}\left(1-2 \varphi \frac{k-1}{\chi}\right)=0, \\
\Lambda=r \chi(k+1)-2 r \varphi(k-1)-(k-1)^{2}(\chi+\varphi) .
\end{gathered}
$$

This equation describes the combustion process as a mechanical oscillating system with friction, wherein a small perturbation surface temperature $\delta \theta_{\mathrm{s}}$ can be described by the ordinary differential equation of the second order (Sabdenov, K.O., 1993, Sabdenov, K.O., 2013).

In the case of positive value of the expression in brackets (21) the stability condition is given by the inequality $\Lambda>$ 0 , or

$$
r>\frac{(k-1)^{2}(\chi+\varphi)}{\chi(k+1)-2 \varphi(k-1)} .
$$

This formula is applicable only in cases where the state of burning corresponds to the solid curve $O_{1} O_{2}$ (Fig. 1).

In the absence of heat loss (22) is converted to a known relationship $(\varphi=0$ and $\chi=1)$, obtained in the framework of ZN-theory: $r>(k-1)^{2} /(k+1)$ (Novozhilov, B.V., 1974).

Fig. 2 shows the limits of stable combustion at low values of the parameter $\varphi$. As can be seen, the increase in heat 
losses leads to a narrowing of the stability region.

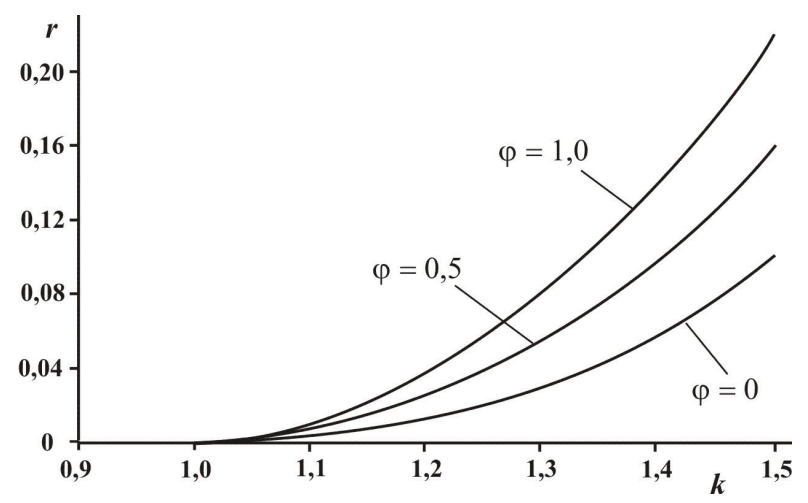

Fig. 2. Changing of combustion stability region at different values of $\varphi$.

It is known (Zenin, A.A., 1980) that with decreasing initial temperature $T_{0}$ for ballistic powders parameter $k$ is reduced. In this case the heat loss from the sides of the propellant sample increases and according to (22) combustion of powder under these conditions can be unstable. Experimental observations (Zenin, A.A., 1980) confirm this: with decreasing initial temperature combustion becomes unstable and becomes a focal and pulsating character. The burning stops for sufficiently deep reduction of $T_{0}$.

These experimental facts can be explained as follows. From (21) follows that the instability arises also in the case if $1-2 \varphi(k-1) / \chi<0$. If this inequality is replaced by an equal sign, it follows, accounting (16), the expression for a critical heat loss coefficient $\varphi_{\text {cr: }}$ :

$$
\varphi_{\mathrm{cr}}=\frac{2 k-1}{4(k-1)^{2}} .
$$

It is clear that for large heat losses $\varphi>\varphi_{\mathrm{cr}}$ instability of steady state can only mean extinction (and then burning of propellant loses oscillatory characteristics). Consequently, condition (22) should be updated by the requirement

$$
k<1+\mathrm{ч} / 2 \varphi=1+\frac{1+\sqrt{1+4 \varphi}}{4 \varphi}
$$

Unfortunately, we were unable to find experimental data for the critical values of the $\varphi_{\mathrm{cr}}$.

In practice, $r<<1$, so that the second condition is essential only for large values of $\varphi$.

Taking a few specific values of $\varphi$, we calculate the limits of the stable combustion in the plane $r k$ with regard to the inequality (23) (Fig. 3).

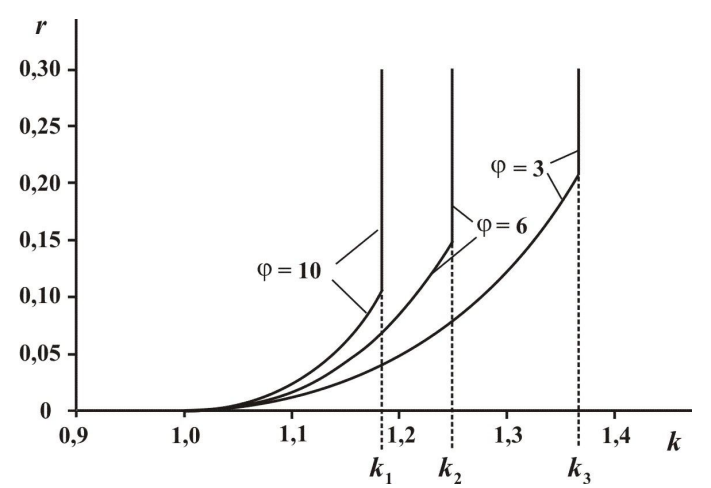

Fig. 3. Boundaries of the regions of stable combustion at high heat losses; $k_{1} \approx 1,18, k_{2} \approx 1,25, k_{3} \approx 1,38$.

Let us give explanations to the possible modes of combustion on this plane. Suppose, for example, $\varphi=6$. Coordinate of the points of intersection of solid and dashed lines $k_{2}=1+\chi / 2 \varphi \approx 1,25$. For the points lying to the left of these lines, the combustion is stable.

For points lying to the right of these lines can be the following: if $k<k_{2}$, then burning is unstable, but an oscillating 
mode can be observed; if $k>k_{2}$ and any $r$, then there is extinction.

\section{Conclusion}

Thus, the proposed theory based on the effective initial temperature does not contradict the ZN-theory. But chances of $T_{0}{ }^{*}$-theory are wider than of $\mathrm{ZN}$-theory. Here to two fundamental differences can be pointed:

1 ) if the results obtained in frames of the $\mathrm{ZN}$-theory, are not limited in conditions of combustion, the $T_{0}{ }^{*}$-theory the stability state of combustion must be placed on the curve (Fig. 1) between points $O_{1} O_{3}$;

2) in $T_{0}{ }^{*}$-theory the condition of an extinction is specified for the case of constant pressure: the effective initial temperature should be less than the minimum initial temperature which is experimentally observed for stationary combustion.

Mathematical calculation within the $T_{0}{ }^{*}$-theory is much simpler compared to the ZN-theory.

As an example, the analysis of combustion stability in the presence of heat loss is performed.

It shows the existence of a critical value of heat transfer coefficient $\varphi_{\mathrm{cr}}$, and if $\varphi>\varphi_{\mathrm{cr}}$ the extinction occurs. With increasing $\varphi$ the steady burning is possible near $k=1$ or less.

\section{References}

Apte, S. and Yang, V., Unsteady flow evolution and combustion dynamics of homogeneous solid propellant in rocket motors, Combustion and Flame, Vol. 131 (2002), pp. 110-131.

Beckstead, M.W., Derr, R.L. and Price, C.F., A model of composite solid-propellant combustion based on multiple flames, AIAA Journal, Vol. 8, Issue 12 (1970), pp. 2200-2207.

Beckstead, M.W., Puduppakkam, K., Thakre, P. and Yang, V., Modeling of combustion and ignition of solid-propellant ingredients, Progress in Energy and Combustion Science, Vol. 33, Issue 6 (2007), pp. 497-551.

Cai, W., Ma, F. and Yang, V., Two-phase vorticoacoustic flow interactions in solid-propellant rocket motors, Journal of Propulsion and Power, Vol. 19, No. 3 (2003), pp. 385-396.

Denison, M.R. and Baum, E.A., A simplified model of unstable burning in solid propellants, ARS Journal, Vol. 31 (1961), pp. 1112-1122.

Greatrix, D. R., Model for prediction of negative and positive erosive burning, Canadian Aeronautics and Space Journal, Vol. 53, No.1 (2007), pp. 13-21.

Krier, H., T'ien, J.S., Sirignano, W.A. and Summerfield, M., Nonsteady burning phenomena of solid propellants: Theory and Experiments, AIAA Journal, Vol. 6 (1968), pp. 278-285.

Mukunda, H.S., A Comprehensive theory of erosive burning in solid rocket propellants, Combustion Science and Technology, Vol. 18, Issue 3-4 (1978), pp. 105-118.

Novozhilov, B.V., Nonstationary combustion of solid rocket fuels. Nauka, Moscow (in USSR), 1973 (Translation AFSC FTD-MD-24-317-74).

Novozhilov, B.V., Theory of nonsteady burning and combustion stability of solid propellants by the Zeldovich Novozhilov method, Combustion Stability of Solid-Propellant, L. De Luca, E.W. Price, and M. Summerfield (Eds.), Progress in Astronautics and Aeronautics, V. 143. Washington: AIAA (1990), pp. 601-641.

Roh, T.-S., Tseng, I.-S. and Yang, V., Effect of acoustic oscillation on flame dynamics of homogeneous propellants in rocket motors, Journal Propulsion and Power, Vol. 11, No. 4 (1995), pp. 640-650.

Sabdenov, K.O., Unsteady combustion in terms of the theory dynamics systems, Chemical Physics Reports, Vol. 12, No.3 (1993), pp. 419-424.

Sabdenov, K.O., On the new approach in theoretical research of non-stationary processes in burning of solid fuel, Theory of Combustion of Powder and Explosives, Nova Science Publication Incorporated (1996), pp. 203-213.

Sabdenov, K.O., On the threshold nature of erosive burning, Combustion, Explosion and Shock Waves, Vol. 44, No.3 (2008), pp. 300-309.

Sabdenov, K.O. and Erzada Maira, Mechanism of the negative erosion effect, Combustion, Explosion and Shock Waves, Vol. 48, No. 3 (2013), pp. 273-282.

Sabdenov, K.O., Nonstability combustion of solid rocket fuels (in Russian), (2013), Academic Publishing GmbH \& Co. KG, Saarbrucken, Germany.

Sandau R., Roeser H.-P. and Valenzuela A., Small Satellites for Earth Observation, (2008), Springer. 
Srinivasan, K., Narayanan, S. and Sharma, O.P., Numerical studies on erosive burning in cylindrical solid propellant grain, Heat Mass Transfer, Vol. 44 (2008), pp. 579-585.

The Role of Small Satellites in NASA and NOAA Earth Observation Programs (online), available from $<$ http://www.nap.edu/catalog/9817.html>, (accessed on 14 June, 2014).

Vilyunov, V.N. and Sabdenov, K.O., To phenomenological theory of unsteady burning, Chemical Physics Reports, Vol. 11, No.3 (1992), pp. 415-423.

Wang, X., Jackson, T.L. and Massa, L., Numerical simulation of heterogeneous propellant combustion by a level set method, Combustion Theory and Modeling, Vol. 8, Issue 2 (2004), pp. 227-254.

Willcox, M.A., Brewster, M.Q., Tang, K.C., Stewart, D.S. and Kuznetzov, I., Solid rocket motor internal ballistics simulation using three-dimensional grain burnback, Journal of Propulsion and Power, Vol. 23, No.3 (2007), pp. 575-584.

Zarko. V.E. and Kiskin A.B., Numerical Modeling of Nonsteady Powder Combustion Under the Action of a Light Flux, Combustion, Explosion, and Shock Waves, Vol. 16, No. 6 (1980), pp. 650-654.

Zarko V.E., Simonenko V.N. and Kiskin A.B., Radiation-Driven, Transient Burning: Experimental Results, Nonsteady Burning and Combustion Stability of Solid Propellants. De Luca, L., Price, E.W., Summerfield, M. (Eds.) Progress in Astronautics and Aeronautics, (1987), Vol. 140. Washington: AIAA (1987), pp. 363-398.

Zarko, V.E. and Gusachenko, L.K., Critical revive of phenomenological models for steadying transient combustion of solid propellants, International Journal of Spray and Combustion Dynamics, Vol. 2, No.2 (2010), pp. 151-168.

Zeldovich, Ya. B., On the theory of combustion of gunpowder and explosives, Journal of Experimental and Theoretical Physics (in USSR), Vol. 12, No. 11, 12 (1942), pp. 24-32.

Zenin, A.A., Processes in zones of burning of ballasting gunpowder, (1980), Atomizdat, Moscow (in USSR). 